\title{
Erratum: Analysis of intronic and exonic reads in RNA-seq data characterizes transcriptional and post-transcriptional regulation
}

Dimos Gaidatzis, Lukas Burger, Maria Florescu \& Michael B Stadler

Nat. Biotechnol. 33, 722-729 (2015); published online 22 June 2015; corrected after print 8 July 2015

In the version of this article initially published online, several errors appeared in the HTML version. In the section "EISA recovers the role of transcription during neurogenesis," the expression " $(\mathrm{t} 1 / 2=1 / \alpha)$ " should have read " $\mathrm{t} 1 / 2 \sim 1 / \alpha)$ " in the sentence "mRNA half-life, on the other hand, is inversely proportional to the degradation rate $(\mathrm{t} 1 / 2=1 / \alpha)$." In the Online Methods, "Analysis of circadian dynamics data sets," the symbol "<-” was given as " $\leq$ " in two cases and as " $\leq<$ " in one case; the formulas "coeffs $\leq \operatorname{lm}\left(\mathrm{y} \sim \operatorname{cbind}\left(\cos \left(\mathrm{w}^{\star} \mathrm{t}\right),-\sin \left(\mathrm{w}^{\star} \mathrm{t}\right)\right)\right) \$ \operatorname{coefficients";~"C~} \leq<\operatorname{sqrt}(\operatorname{coeffs}[2] \wedge 2+$ coeffs[3]^2)"; " $\phi \leq$ atan2(coeffs[3], coeffs[2])" should have been "coeffs $<-\operatorname{lm}\left(\mathrm{y} \sim \operatorname{cbind}\left(\cos \left(\mathrm{w}^{\star} \mathrm{t}\right),-\sin \left(\mathrm{w}^{\star} \mathrm{t}\right)\right)\right) \$ \operatorname{coefficients";~"C<-~sqrt}(\operatorname{coeffs}[2] \wedge 2$ $\left.+\operatorname{coeffs}[3]^{\wedge} 2\right)^{\prime} ;$; $\phi<-$ atan2(coeffs[3],coeffs[2])." In addition, the corresponding authors are Dimos Gaidatzis, Lukas Burger and Michael Stadler, rather than Dimos Gaidatzis, Lukas Burger and Maria Florescu.The errors have been corrected in HTML version of this article.

\section{Erratum: Share and share alike}

Samuel K Sia \& Matthew P Owens

Nat. Biotechnol. 33, 1224-1228 (2015); published online 9 December 2015; corrected after print 13 January 2016

In the version of this article initially published, in Box 2, the company name Celmatix was misspelled twice as Celmatrix. The errors have been corrected in the HTML and PDF versions of the article.

\section{Corrigendum: Increasing the efficiency of precise genome editing with CRISPR-Cas9 by inhibition of nonhomologous end joining}

Takeshi Maruyama, Stephanie K Dougan, Matthias C Truttmann, Angelina M Bilate, Jessica R Ingram \& Hidde L Ploegh Nat. Biotechnol. 33, 538-542 (2015); published online 23 March 2015; corrected online 16 April 2015

In the version of this article initially published online, the received date of the paper was given as 17 October 2014 ; the correct date is 11 July 2014. On p.4, left column, second paragraph, $1 \mu \mathrm{M}$ Scr7 should have been $1 \mathrm{mM}$ Scr7; in Online Methods, second paragraph, Cas9 mRNA, sgRNA and template oligos were erroneously given in $\mathrm{ng} / \mathrm{ml}$ rather than $\mathrm{ng} / \mu \mathrm{l}$. A technique was misidentified as ChIP; in all cases it should be "chip." The possibility of using Scr7 as a means of enhancing homology-directed repair was recently also mentioned in a review article that was published while our manuscript was under review. A reference to this article has been added to the manuscript. Finally, the $P$ values in Table 1 for Kell and Lgkc were reversed and should be ${ }^{\star} P<0.05$ (Igkc), ${ }^{\star *} P<0.005$ (Kell) and not ${ }^{\star} P<0.05$ (Kell), ${ }^{\star * \star} P<0.005$ (Igkc). The errors have been corrected for the print, PDF and HTML versions of this article. 